\title{
Prediction of Multiphase Alternative of Acute Disseminated Encephalomyelitis Course Development
}

\author{
Iryna Lobanova, Olena Myalovitska \\ Department of Neurology, National Medical University, Kiev City, Ukraine \\ Email: lobanovanmu@gmail.com
}

Received 15 February 2014; revised 15 March 2014; accepted 3 April 2014

Copyright () 2014 by authors and Scientific Research Publishing Inc.

This work is licensed under the Creative Commons Attribution International License (CC BY). http://creativecommons.org/licenses/by/4.0/

(c) $\underset{\mathrm{EY}}{\mathrm{EP}}$ Open Access

\begin{abstract}
Prediction of development of the multi-phase alternative for the course inherent to acute disseminated encephalomyelitis (ADEM) is of great clinical importance, as it enables timely determination of the treatment tactics as well as volume of respective therapeutic interventions. This work is aimed at ascertaining the prognostic factors that determine the risk of development of the multi-phase course in disseminated encephalomyelitis. We have examined 101 patients with the diagnosis ADEM, namely: 28 men and 73 women in the age from 17 up to 53 years (average value $31.7 \pm 1.01$ years). To ascertain the prognostic meaning of clinic-paraclinic indices corresponding to patients with ADEM, we estimated the cumulative part of absence of relapses in the group of patients by using the Kaplan-Meyer method with estimating the Fisher criterion and using the most important clinic-paraclinic data. Development of the multiphase course in ADEM is reliably related to the following prognostic signs: changes in the neurologic status of patients with ADEM, degree of disability in accord with the EDSS scale as well as sizes of demyelination focuses determined using MRT. Criteria for congenial prediction in disease development with delayed appearance of ADEM relapses in the form of the multi-phase course are as follows: domination of motor impairments over coordinative impairments in neurological status, slight degree (in EDSS scale) of disability and small sizes (up to $4 \mathrm{~mm}$ ) of demyelination focuses (MRT data). Our analysis of the main clinic-paraclinic indices obtained using the Kaplan-Meyer method indicates reliability of results and enables us to find a number of important prognostic criteria for appearance of the multiphase course in ADEM.
\end{abstract}

\section{Keywords}

Acute Disseminated Encephalomyelitis, Prediction, Kaplan-Meyer Method 


\section{Introduction}

The most often, ADEM is characterized by the single-phase course accompanied by considerable variations concerning the duration of disease and convalescence. However, also there are relapses of ADEM known already from 1932 year due to van Bogaert who published the paper "ADEM with relapses" [1]. ADEM relapses can be considered as a multi-phases course of this disease or its transformation into multiple sclerosis (see, e.g., the works [2]-[7]).

ADEM relapses are observed with the following frequencies: in one case from 18 ones for this disease (5.5\%) [8], in 4 of 31 (13\%) [9] [10], in 24 of 132 (18\%) [11], in 7 of 35 (20\%) [12], in 9 of 42 (21\%) [13], in 5 of 21 (24\%) [14].

Appearance of new clinic symptoms in three months after initial signs of this disease is considered as a relapse of ADEM. In the case of this disease relapse, the pathological process comprises new parts of brain and/or spinal cord (which is usually confirmed by clinical investigations and neurovisual methods).

If the relapse appears in a short time interval after initial signs and is combined with further infection or cancelled hormonal therapy, the term multiphase disseminated encephalomyelitis (MDEM) should be used [15] [16].

In opinion of researchers, MDEM is characterized by poly-symptomatic presentations of this disease, availability of demyelination focuses in MRT data mainly in subcortical parts of brain, in less degree located periventricularly, with total or partial disappearance of focuses within the convalescent period [17]. The multiphase course of disseminated encephalomyelitis can be diagnosed in the case of disease relapse appearance at least 3 months after its initial presentation [14] [16]-[18]. Appearance of new clinic symptoms and new focuses in MRT data 12 to 18 months after the primary episode of the disease is indicative of its possible transformation into multiple sclerosis [19].

In literature there is available information about possible options in the course of ADEM. The results of observations aimed at the course of this disease for 40 patients with the ADEM diagnosis show that 14 (35\%) of them demonstrate developed clinically confirmed multiple sclerosis one year after the primary ADEM episode [20]. Another investigation showed that 5 (23.8\%) from 21 patients demonstrated relapses of disseminated encephalomyelitis, and 2 (9.5\%) of these 21 patients had multiple sclerosis. The recent investigations showed that 4 (13\%) of 31 patients with ADEM had manifestations of ADEM relapses, while 10 (40\%) of 25 patients had clinically confirmed multiple sclerosis [21]. As it follows from the data of Schwarz, S., et al.'s [20] [22], developed multiple sclerosis was found in 14 (35\%) of 40 patients in 38-month period of observations (which was confirmed using the Poser criteria). In the Alan investigations, 13 (33.3\%) of 39 patients faced the replaces of demyelination disease [9] [21]. For the long period of observations (8 years), only one patient with ADEM of 11 showed new focuses of demyelination in MRT data [20].

The investigations performed in France indicate that 57\% patients with ADEM can acquire multiple sclerosis in 2.9 years, in the average. Investigation of 48 patients with ADEM showed that 10 ones (20.8\%) acquire multiple sclerosis in 2.36 years, while 13 (27\%) in 5.64 years [12].

From the viewpoint of clinical neurology, not only the frequency of the multiphase option in the course of ADEM but also determination of prognostic risk factors that cause it are of great importance too. Prediction of the multiphase course of ADEM and its transformation into disseminated sclerosis is of great importance since it enables to determine the tactics treating the patients and volume of therapeutic measures.

The purpose of this research was to determine the prognostic risk factors of development of the multiphase course in disseminated encephalomyelitis.

\section{Methods}

We have examined 101 patients with the diagnosis ADEM, namely: 28 men and 73 women in the age from 17 up to 53 years (average age value $31.7 \pm 1.01$ years). All the patients were investigated using magnetic-resonance tomography (MRT) investigations of brain and/or spinal cord as well as estimated the disability degree in accord with the Kurztke Expanded Disability Status Score (EDSS) scale [23].

All the patients were under observation for 3 years. If during this period we could not mark any relapse of demyelination disease, we interpreted it as the single-phase type of the ADEM course. In the case when there arose disease relapses that, from the clinical viewpoint and after neuro-visual patient examination, had signs of disseminated encephalomyelitis, we considered it as the multiphase option of its course. In the cases of clinically 
confirmed multiple sclerosis (in accord with the McDonald criteria [24], we interpreted it as a transfer of ADEM into multiple sclerosis.

To ascertain the prognostic meaning of clinic-paraclinic indices for patients with ADEM, we estimated the cumulative part of the absence of relapses by using the Kaplan-Meyer method with the Fisher criterion and the most important clinic-paraclinic data [25] [26].

\section{Results}

The total prediction of cumulative parts for ADEM relapses in the form of its multi-phase course is adduced in Figure 1.

As seen from Figure 1, 10\% (cumulative part is equal to 0.1) of patients with ADEM show the relapse 4 months after first signs of this disease, 25\% (cumulative part-0.25)—after the half-year (6 months), 50\% (cumulative part-0.5) —after one year (12 months), 75\% (cumulative part-0.75)—after 1.5 year (18 months) of the period for observation. The cumulative part of patients without relapses for the first 6 months was 0.75 (i.e., $75 \%$ of all the patients), for 12 months -0.5 ( $50 \%$ of patients), for 24 months -0.05 ( $5 \%$ of patients).

The dynamics of decay in the cumulative part of patients with ADEM but without relapses in the multiphase course within the three year period of observations is depicted in Figure 2.

As seen in Figure 2, in case of patients with the multiphase course of disseminated encephalomyelitis, the most pronounced decay in the cumulative part of patients without relapses was observed during the first year of observations (50\%), somewhat less—during the second year (45\%), and the least one—during the third year (5\%).

Thus, the frequency of relapses changed in dependence on the term of observations for three year period, and it was the highest during the first year and the lowest during the third year.

The prognostic estimate of development inherent to the multiphase course of ADE in dependence on the age is depicted in Figure 3.

As seen in Figure 3, the best indices of replace absence were observed in patients of the age 39 to 40 years, however the statistical difference is not reliable $(p=0.067(>0.05))$.

Prediction of development of the multiphase course in ADEM in dependence on gender did not find any reliable difference between the three-year absence of replaces in men and women, but one could observe a tendency of increasing the cumulative part in women $(p=0.07213,(>0.05))$ (Figure 4).

Thus, one can draw a conclusion that such prognostic signs as age and gender have no reliable influence on development of relapses in ADEM in the form of its multiphase course.

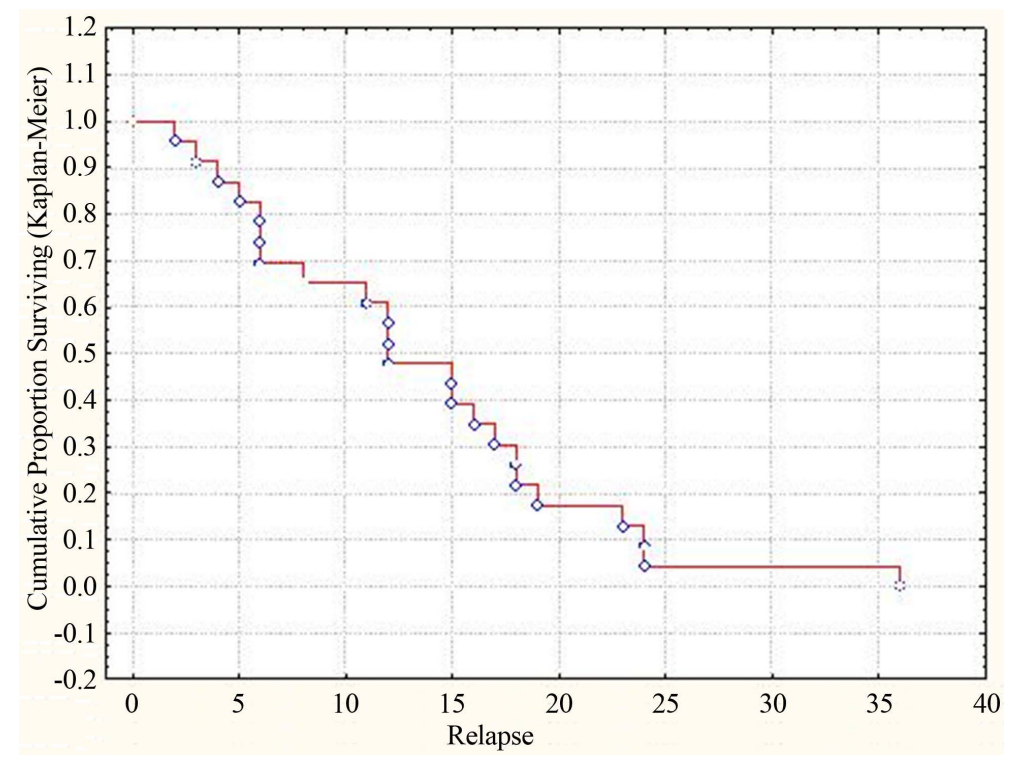

Figure 1. Dynamics of the cumulative part of patients with ADEM without development of the multiphase course for the three year period of observations (by the Kaplan-Meyer method). 


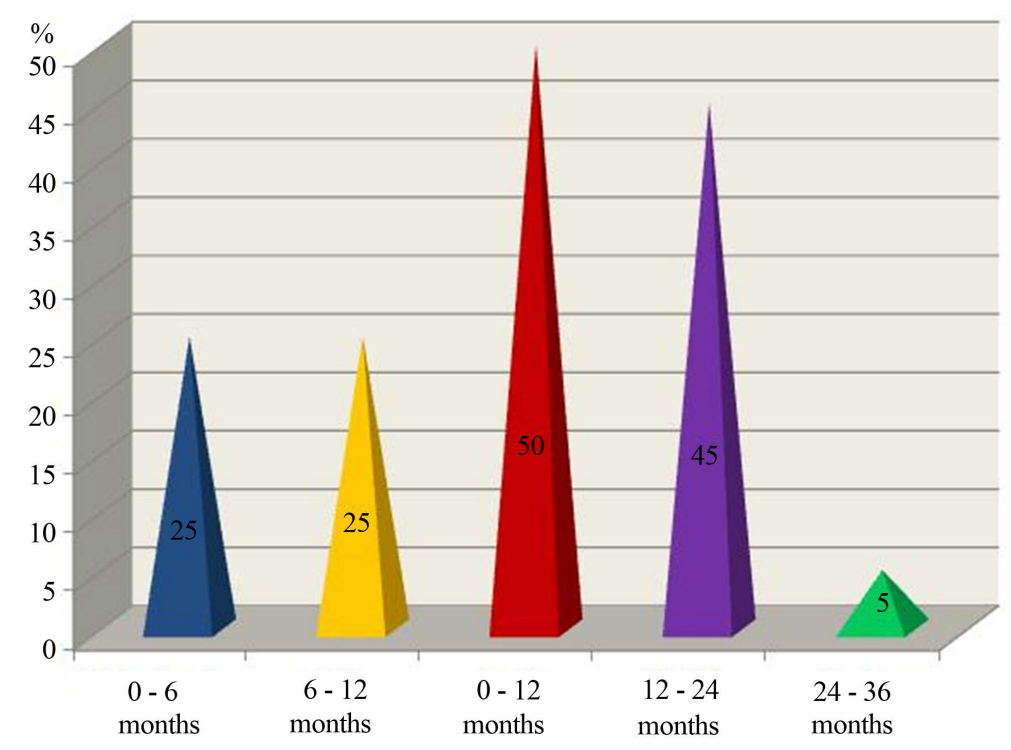

Figure 2. Dynamics of decrease in the cumulative part of patients with ADEM but without development of the multiphase course in this disease for the three year period of observations (in \%).

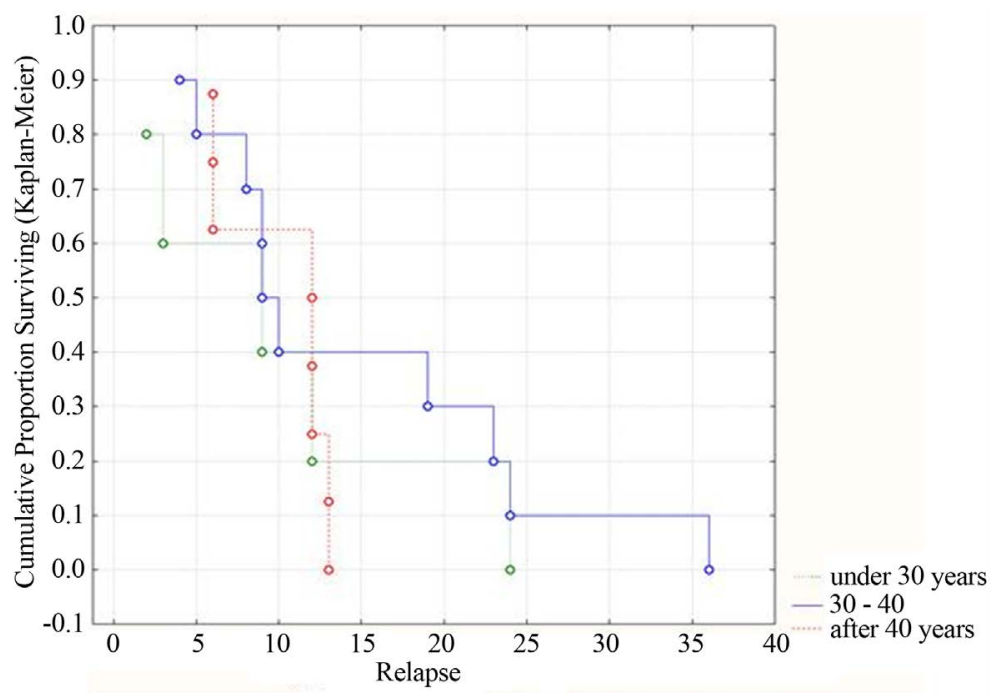

Figure 3. Dynamics of the cumulative part of patients without relapses by separate age groups (using the Kaplan-Meyer method).

Availability of changes in the neurologic status in the form of coordinative impairments is combined with development of MDEM in earlier terms of observation as compared with neurologic deficiencies in the form of motor impairments, which is an important prognostic sign $(\mathrm{p}=0.02803,(<0.05))$ (Figure 5).

The complex estimate of the status of patients with ADEM by the EDSS scale and its relation with the term of appearance of MDEM signs are depicted in Figure 6.

As seen in Figure 6, the multiphase course of ADEM arose later (beginning from 9 months) in patients with a slight disability degree by the EDSS scale, as compared to those with a medium or heavy disability degrees $(\mathrm{p}=$ 0.00141, $(<0.001))$.

Thus, availability of a slight disability degree by the above scale corresponds to a more positive course of ADEM, which is manifested in the increased cumulative part of patients without relapses as well as term of MDEM emergence.

When analyzing the MRT data taken from patients with ADEM, we ascertained that availability of demyeli- 


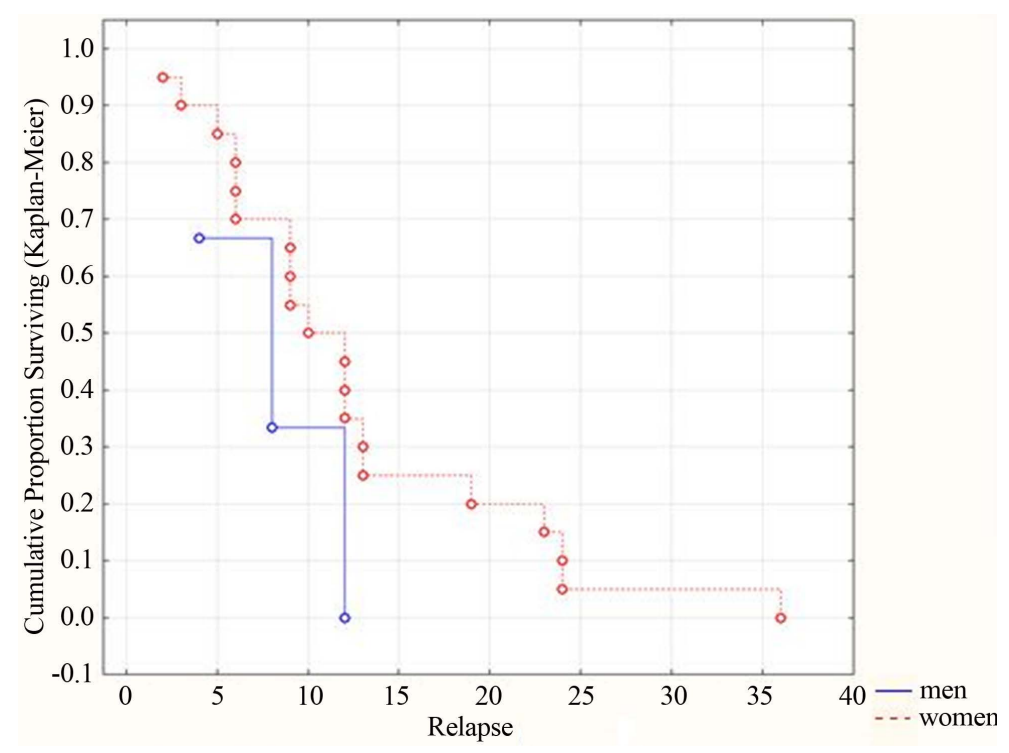

Figure 4. Dynamics of the cumulative part of patients without relapses in dependence on their gender (by the Kaplan-Meyer method).

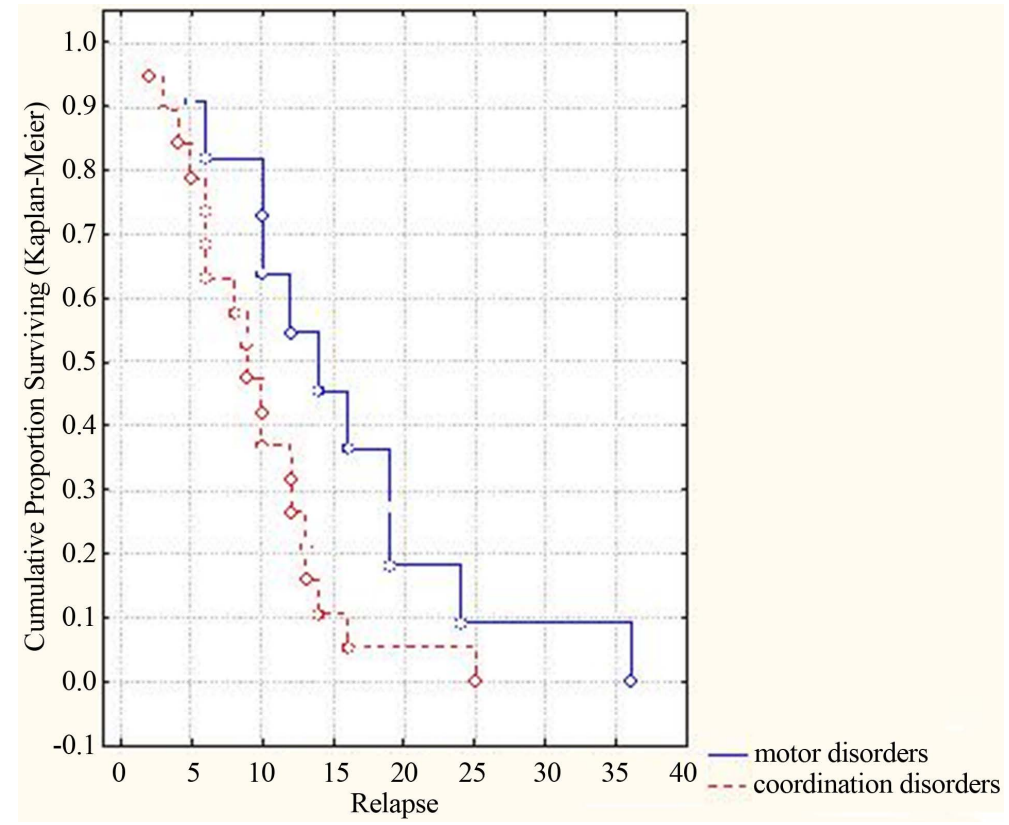

Figure 5. Dynamics of the cumulative part of patients without relapses in dependence on changes in the neurologic status (by Kaplan-Meyer).

nation focuses with various medium sizes makes an essential effect on prediction of the ADEM course, in particular its multiphase option, which is adduced in Figure 7.

Thus, the smaller is the medium size of demyelination focuses, the slower is development of the multiphase course in this disease (replaces begin to arise after the first year of observations $(p=0.02179,(<0.05))$.

\section{Conclusion}

Thus, our analysis of the main clinic-paraclinic indices by the Kaplan-Meyer method proved to be reliable and enabled us to find out a number of important prognostic criteria of the multiphase course appearance in ADEM. The reliable influence on development of the multiphase course in ADEM is related to such prognostic signs as 


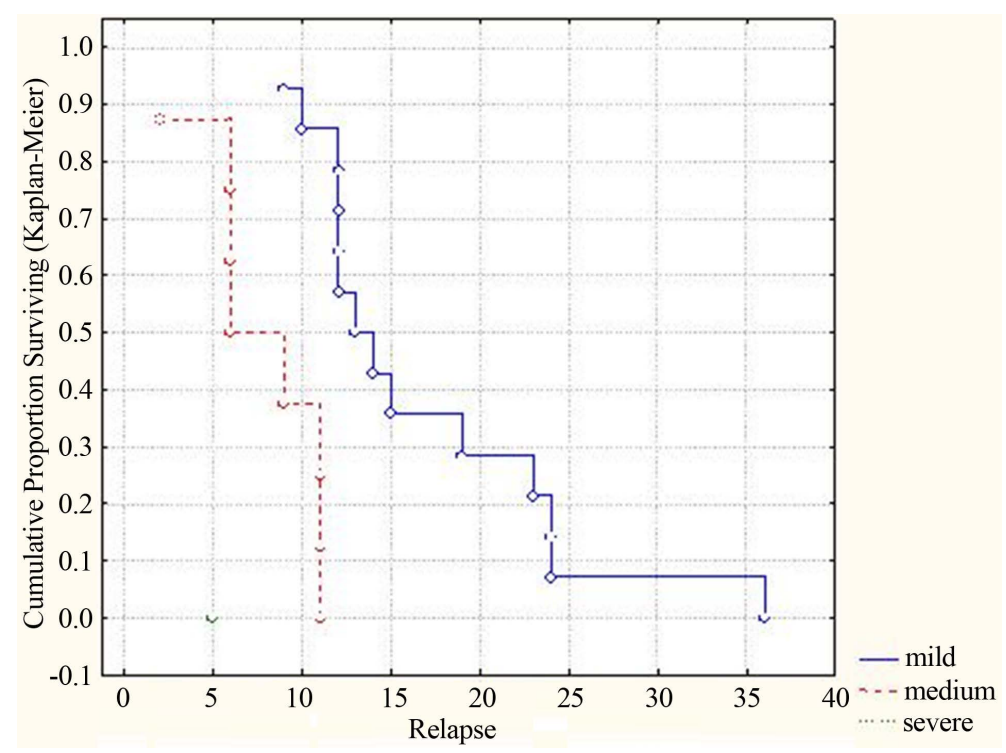

Figure 6. Dynamics of the cumulative part of patients without relapses by the disability degree in accord with the EDSS scale (by Kaplan-Meyer).

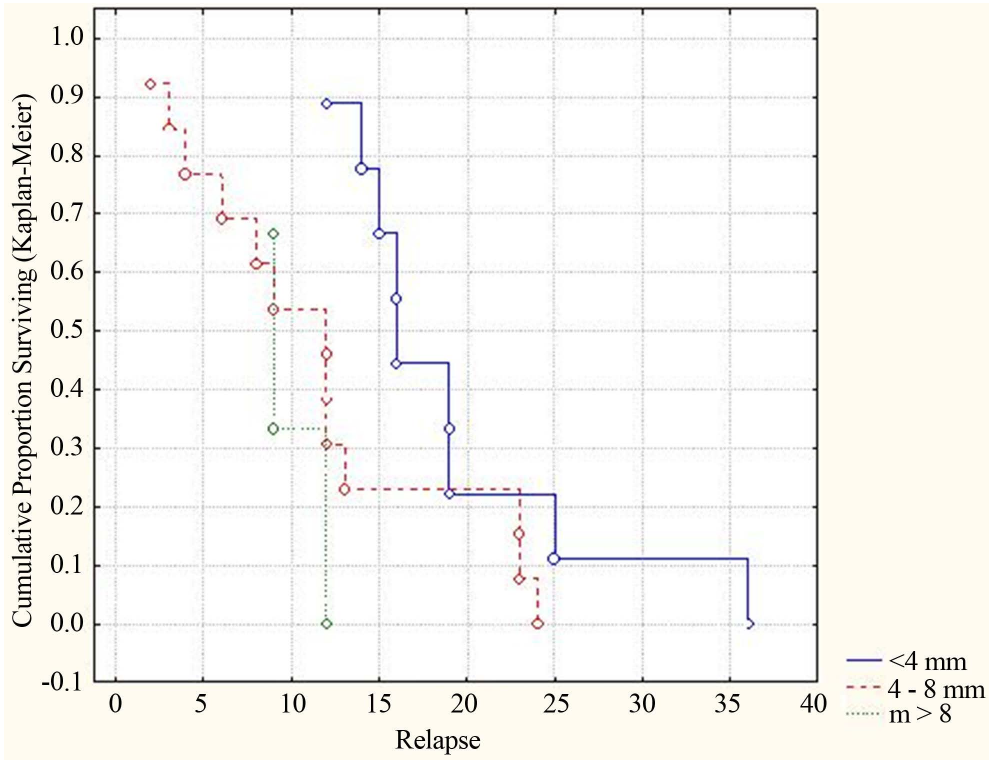

Figure 7. Dynamics of the cumulative part of patients without relapses by sizes of demyelination focuses in accord with MRT data (by Kaplan-Meyer).

changes of the neurologic status in patients with ADEM, disability degree by the EDSS scale as well as size of demyelination focuses in accord with MRT data. The criteria for favorable prediction in this disease with later development of ADEM replaces in the form of multiphase course are domination of motor impairments over the coordination ones in the neurologic status, slight degree of disability by the EDSS scale, and small size (up to 4 $\mathrm{mm}$ ) of demyelination focuses (MRT data).

\section{References}

[1] Tenembaum, S. (2008) Disseminated Encephalomyelitis in Children. Clinical Neurology and Neurosurgery, 110, 928-938. http://dx.doi.org/10.1016/j.clineuro.2007.12.018

[2] Brass, S.D. (2003) Multiple Sclerosis and Acute Disseminated Encephalomyelitis in Childhood. Pediatric Neurology, 


\section{9, 227-231. http://dx.doi.org/10.1016/S0887-8994(03)00235-2}

[3] McGovern, R.A. and DiMario, F.J. (2003) Acute Disseminated Encephalomyelitis: A Retrospective Pediatric Serie. Annals of Neurology, 54, 127-129.

[4] Neuteboom, R.F, Catsman-Berrevoets, C.E. and Hintzen, R.Q. (2007) Multiple Sclerosis in Children. Nederlands Tijdschrift voor Geneeskunde, 151, 1464-1468.

[5] Pohl, D, Hennemuth, I. and Kries, R. (2007) Paediatric Multiple Sclerosis and Acute Disseminated Encephalomyelitis in Germany: Results of a Nationwide Survey. European Journal of Pediatrics, 166, 405-412. http://dx.doi.org/10.1007/s00431-006-0249-2

[6] Toshiyuki, O. and Shunsaku, H. (2004) Reccurence of Acute Disseminated Encephalomyelitis after a 12-Year Symptom-Free Interval. Interval Medicine, 43, 746-749. http://dx.doi.org/10.2169/internalmedicine.43.746

[7] Tur, C, Téllez, N. and Rovira, A. (2008) Acute Disseminated Encephalomyelitis: Study of Factors Involved in a Possible Development towards Multiple Sclerosis. Neurologia, 23, 546-554.

[8] Hung, K.L. (2001) The Spectrum of Postinfectious Encephalomyelitis. Brain \& Development, 23, 42-45. http://dx.doi.org/10.1016/S0387-7604(00)00197-2

[9] Anlar, B. (2003) Acute Disseminated Encephalomyelitis in Children: Outcome and Prognosis. Neuropediatrics, 34, 194-199. http://dx.doi.org/10.1055/s-2003-42208

[10] Huynh, W, Cordato, D.J. and Kehdi, E. (2008) Post-Vaccination Encephalomyelitis: Literature Review and Illustrative Case. Journal of Clinical Neuroscience, 15, 1315-1322. http://dx.doi.org/10.1016/j.jocn.2008.05.002

[11] Mikaeloff, Y, Caridade, G, Husson, B, et al. (2007) Acute Disseminated Encephalomyelitis Cohort Study: Prognostic Factors for Relapse. European Journal of Paediatric Neurology, 11, 90-95. http://dx.doi.org/10.1016/j.ejpn.2006.11.007

[12] Dale, R.C, Sousa, C. and Chong, W.K. (2000) Acute Disseminated Encephalomyelitis, Multiphasic Disseminated Encephalomyelitis and Multiple Sclerosis in Children. Brain, 123, 2407-2224. http://dx.doi.org/10.1093/brain/123.12.2407

[13] Leake, J.A. (2004) Acute Disseminated Encephalomyelitis in Childhood: Epidemiologic, Clinical and Laboratory Features. The Pediatric Infectious Disease Journal, 23, 756-764. http://dx.doi.org/10.1097/01.inf.0000133048.75452.dd

[14] Cohen, O. (2001) Recurrence of Acute Disseminated Encephalomyelitis at the Previously Affected Brain Site. Archives of Neurology, 58, 797-801. http://dx.doi.org/10.1001/archneur.58.5.797

[15] Dale, R.C. and Branson, J.A. (2005) Acute Disseminated Encephalomyelitis or Multiple Sclerosis: Can the Initial Presentation Help in Establishing a Correct Diagnosis. Archives of Disease in Childhood, 90, 636-639. http://dx.doi.org/10.1136/adc.2004.062935

[16] Dale, R.C. and Pillai, S.C. (2007) Early Relapse Risk after a First CNS Inflammatory Demyelination Episode: Examining International Consensus Definitions. Developmental Medicine \& Child Neurology, 49, 887-893. http://dx.doi.org/10.1111/j.1469-8749.2007.00887.x

[17] Divya, S.K., Mrlvin, J.J. and Sanjeev, V.K. (2005) Acute Disseminated Encephalomyelitis in Children: Discordand Neurologic and Neuroimaging Abnormalities and Response to Plasmapheresis. Pediatrics, 166, 431-436.

[18] Tenembaum, S., Chamoles, N. and Fejerman, N. (2002) Acute Disseminated Encephalomyelitis: A Long-Term Follow-Up Study of 84 Pediatric Patients. Neurology, 22, 1224-1231. http://dx.doi.org/10.1212/WNL.59.8.1224

[19] Kanter, D.S., Horensky, D. and Sperling, R.A. (1995) Plasmapheresis in Fulminant Acute Disseminated Encephalomyelitis. Neurology, 45, 824-827. http://dx.doi.org/10.1212/WNL.45.4.824

[20] Murthy, J.M. (2002) Acute Disseminated Encephalomyelitis. Neurology India, 50, 238-243.

[21] Morimatsu, M. (2004) Recurrent ADEM or MS? Internal Medicine, 43, 647-648. http://dx.doi.org/10.2169/internalmedicine.43.647

[22] Schwaz, S. (2001) Acute Disseminated Encephalomyelitis: A Follow-Up Study of 40 Patients. Neurology, 25, 13831318.

[23] Kurtzke, J.F. (1983) Rating Neurological Impairment in Multiple Sclerosis: An Expanded Disability Status Scale (EDSS). Neurology, 3, 1444-1452. http://dx.doi.org/10.1212/WNL.33.11.1444

[24] Polman, C.H., Reingold, S.C., Edan, G., et al. (2005) Diagnostic Criteria for Multiple Sclerosis: 2005 Revisions to the “McDonald Criteria”. Annals of Neurology, 58, 840-846. http://dx.doi.org/10.1002/ana.20703

[25] Kaplan, E.L. and Meier, P. (1958) Nonparametric Estimation from Incomplete Observations. Journal of the American Statistical Association, 53, 457-481. http://dx.doi.org/10.1080/01621459.1958.10501452

[26] Berwick, V., Cheek, L. and Ball, J. (2004) Statistics Review 12: Survival Analysis. Critical Care, 8, 389-394. http://dx.doi.org/10.1186/cc2955 